\title{
Late-onset papilledema following spinal injury. Case report
}

A Catz MD, ${ }^{1,5,6}$ I Appel MD, ${ }^{2,5}$ I Reider-Grosswasser MD ${ }^{3,5}$ Z Grosswasser MD, ${ }^{1,5}$ L Mendelson MD, ${ }^{1,5}$ R Gepstein $\mathrm{MD}^{4,5}$

${ }^{1}$ Spinal and Brain Injuries Departments Loewenstein Rehabilitation Hospital, 278 Achuza Street, PO Box 3, Ra'anana 43100; ${ }^{2}$ Ophthalmology Department, Hasharon Hospital, Petach-Tikva; ${ }^{3}$ Neuro-Radiological Unit, Ichilov Hospital, Tel-Aviv Medical Center; ${ }^{4}$ Orthopedic Department, Spinal Surgery Unit, Meir Hospital, Kfar-Sava; ${ }^{5}$ Sackler Faculty of Medicine, Tel-Aviv University, Tel-Aviv; ${ }^{6}$ IDF Medical Corps, Israel.

Papilledema, is a known complication of various spinal pathologies. It has, however, been only infrequently reported following spinal injury, and may be overlooked in these cases. Presented herein is a 27 year old male who suffered thoracic and lumbar spinal injuries. Papilledema following mild increase in intracranial pressure (IICP) developed 3 weeks following trauma, and subsided within 8 weeks. The importance of routine repeat ophthalmoscopic examinations following spinal injury to detect changes characteristic of IICP is emphasised.

Keywords: papilledema; increased intracranial pressure (IICP); spinal injury.

\section{Introduction}

Papilledema is not a well known complication of spinal trauma. It has, however, been reported following an increase in intracranial pressure (IICP) in patients with various spinal pathologies, such as schwanomas ${ }^{1}$ or astrocytomas ${ }^{2,3}$ and infections. ${ }^{4}$ Presented herein is a patient with spinal injury complicated by papilledema. The papilledema was diagnosed by routine ophthalmologic examination 3 weeks after the injury was sustained, and resolved within 8 weeks. The importance of repeat ophthalmoscopic examinations in patients with spinal trauma is emphasised.

\section{Case report}

A 27 year old male was hit by a car while bicycling. When first examined the patient was fully alert and denied any loss of consciousness, although he could not recall the accident. Neurological examination revealed incomplete motor and sensory loss below the L2 segment, mainly on the right, with reduced muscle tone and total absence of bladder and bowel control. No neurological deficit was found above the L2 level and the optic fundi were normal on ophthalmoscopy. Spinal xray study showed fractures of the T6-T8, D12, L1 and L3 vertebrae. Computerised tomography (CT) demonstrated multiple fracture lines at various sites of vertebral bodies and neural arches, with displacement of bony fragments into the spinal canal (Fig 1). An L1-L3 laminectomy was performed, exposing cord compression at the L1 level, completely severed dura mater at the L2-L3 level, damaged nerve roots and scattered bony fragments. The cord and roots were decompressed, the severed dura mater was reconstructed and the spine was fused (Fig 2). The postoperative

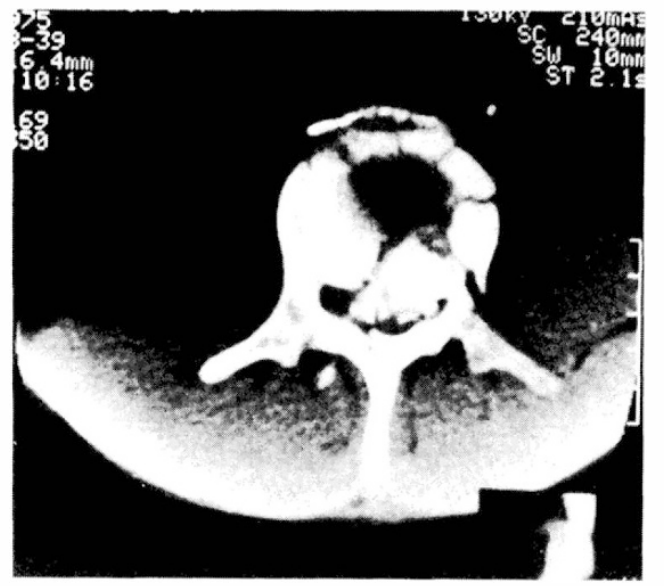

Figure 1 Preoperative CT: burst fracture of L3. Multiple fracture lines in the vertebral body, with posterior displacement of bony fragments into the spinal canal. Linear fractures at various sites of the neural arch. 


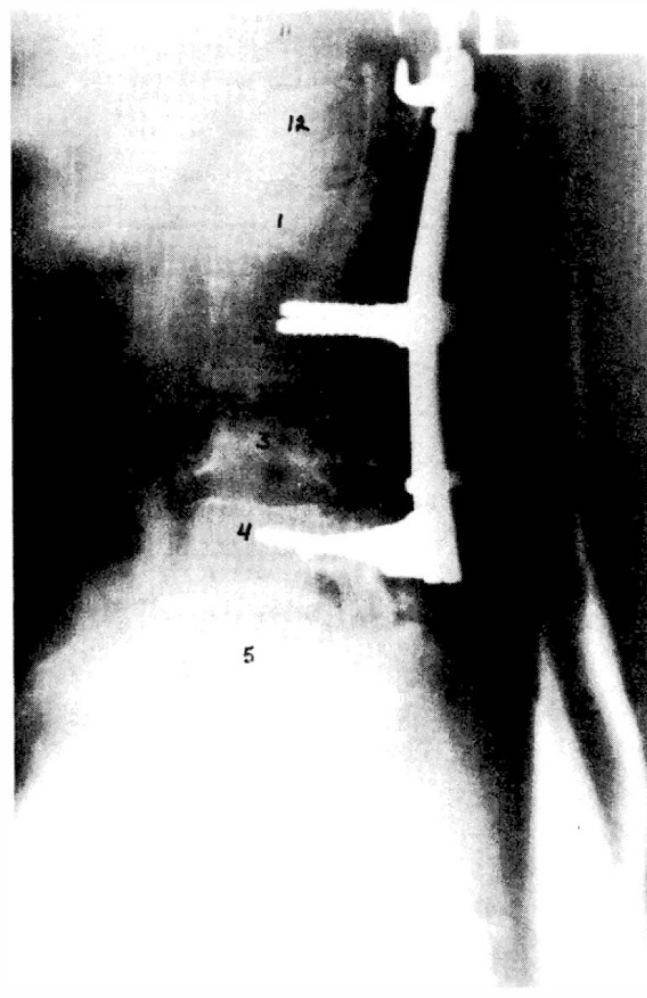

Figure 2 Postoperative lateral xray films of the lumbar spine (T11-L5 as numbered on the vertebral bodies). The spine is stabilised with Cotrel-Dubosset (CD) instrumentation. Note deformity and compression of vertebral bodies T12, L1, L3.

period was uneventful except for the appearance of troublesome headaches. Follow up examinations showed neurological improvement.

Three weeks after the injury, when a rehabilitation programme was started, the patient underwent routine ophthalmoscopic examination which revealed severe papilledema (3-4 diopters), more prominent in the left eye. Oedematous elevation of the nerve heads, complete obliteration of the discs' cups and oedematous blurring of the margins of the discs were observed. There was venous engorgement with loss of venous pulsations, capillary congestion of the discs' heads and haemorrhages in the nerve fibre layer around the discs.

The headaches persisted, but no neurological deterioration was noted; cognitive function remained intact, and visual acuity was not impaired. Brain CT without contrast showed symmetric mild widening of the lateral ventricles including the temporal horns, and of the third ventricle (Fig 3a, b, c); the bifrontal cerebroventricular index (CVI-I described by Hann) ${ }^{5}$ was 0.35 (Fig 3b). The fourth ventricle was normal in size. Moderate symmetrical widening of the cerebral sulci was noted at the periphery of the frontal and temporal lobes. The perimesencephalic cisterns were slightly widened (Fig 3c). The supraventricular sulci, however, were narrow (Fig 3d). In addition, there were small areas of ill-defined low densities in the lower and medial part of the frontal lobes (Fig 3c).

Treatment consisted of dexamethasone ( $24 \mathrm{mg}$ daily gradually tapered off) and acetazolamide ( $750 \mathrm{mg}$ daily). The headaches improved soon after the onset of treatment although the papilledema and the CT findings remained unchanged for about 4 weeks. The papilledema began to subside by the fifth week and disappeared completely within 8 weeks (11 weeks from trauma).

Meanwhile, improvement of the neurological status continued and the patient achieved sphincteric control and unassisted walking. Follow up examinations performed a year later, revealed normal fundi, normal visual acuity and restoration of normal ventricular size on brain CT scans (Fig 4a, b, c). The CVI-I decreased to 0.30 (Fig 4b) and the temporal horns were rather small (Fig 4c). The sulci (mainly those seen in supraventricular cuts) increased in comparison with the first CT study (Fig 4d).

\section{Discussion}

Papilledema has been described following various spinal pathologies but has rarely been reported in association with spinal trauma, when it may remain undetected. ${ }^{1-4}$ The case presented demonstrates the possibility of clinically unexpected late IICP and consequent papilledema following spinal injury. The course of the papilledema was benign, although presumably steroids and acetazolamide administration accelerated its disappearance. Delay in diagnosis could have led to prolongation of the papilledema and a deterioration in visual acuity as a result of optic atrophy. ${ }^{6}$ Therefore, repeat routine ophthalmoscopic examinations are recommended over the course of several weeks after spinal injury.

Hyreh $^{7}$ showed that papilledema can be induced by an increase in pressure in the space around the optic nerve, which com- 
a

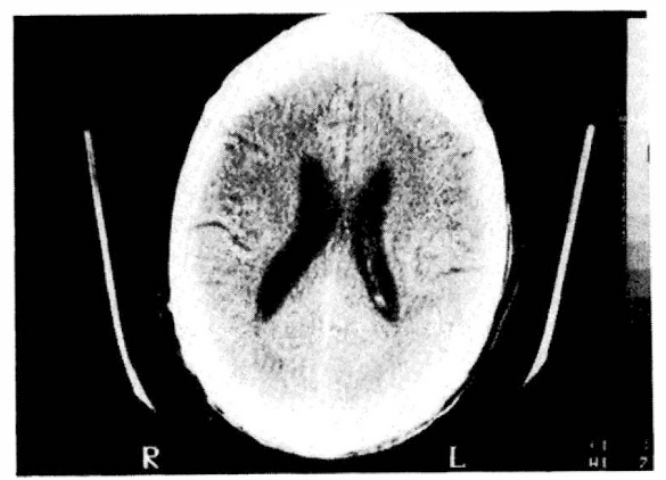

c

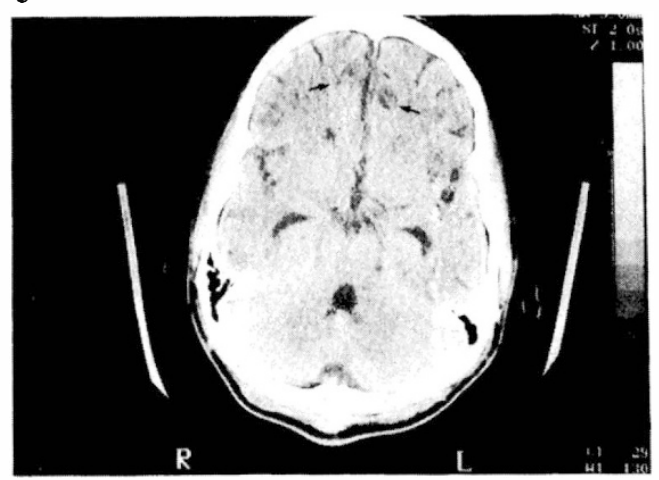

b

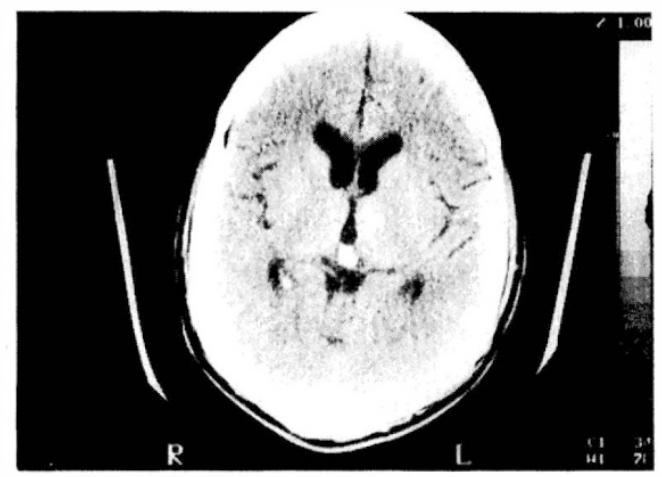

d

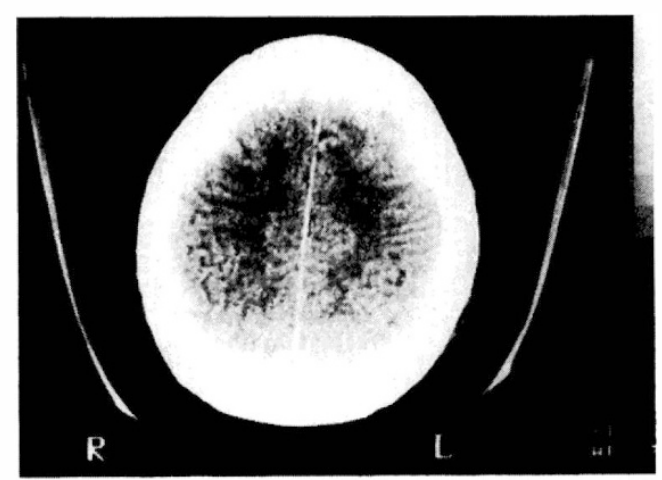

Figure 3 CT of brain: axial cuts (4 weeks after trauma). (a) At the level of the bodies of the lateral ventricles. Symmetric mild to moderate widening of the ventricles and mild widening of the cerebral sulci. (b) At the level of the third ventricle. Mild widening of the frontal horns, third ventricle and cerebral sulci, including the interhemispheric fissure. (c) At the level of the fourth ventricle and lower part of the frontal lobes. Widening of the temporal horns, cerebral sulci, perimesencephalic cisterns and Sylvian fissures. The fourth ventricle is normal in size. There are 2 small parasagittal intracerebral low density areas (arrows). (d) At a supraventricular level. The sulci are narrow.

municates with the subarachnoid space of the brain. This pressure induces stasis of the axoplasmic flow and swelling of the optic nerve and optic disc, with consequent vascular changes of the disc. ${ }^{8,9}$ The brain CT scan appearances are compatible with reversible IICP. The first CT findings combined with the ophthalmoscopic changes suggested this diagnosis, and the last follow up CT study supports it. The ongoing widening of sulci may be associated with mild brain atrophy secondary to the IICP.

The occurrence of IICP in relation to spinal tumours has been attributed to the high protein content of the cerebrospinal fluid (CSF) in affected patients. This increased protein level causes an elevation in colloid-osmotic pressure, which in turn increases CSF volume. ${ }^{4,10}$ The mechanism of IICP induction following spinal injury is unknown, although the increase in protein and blood in the CSF may have a role. Blood and damaged tissue which penetrated the CSF through the dural tears, could have caused IICP in our patient. In a recent report on IICP following spinal injury, a severe wound infection and repeated operations, probably followed by high CSF protein content, were involved. ${ }^{4}$ Damage to the spinal sympathetic outflow may appear to 
a

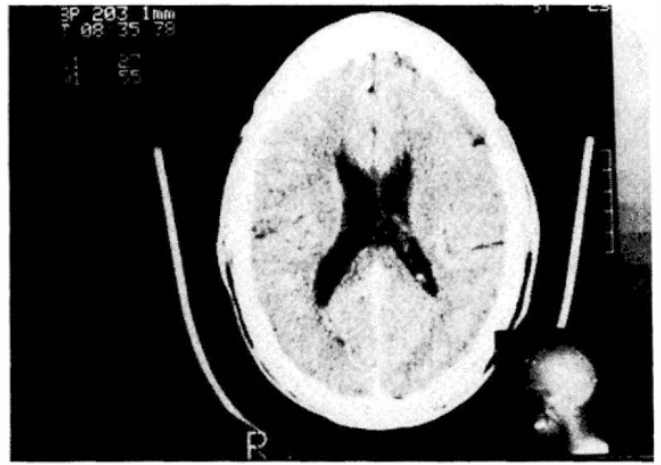

c

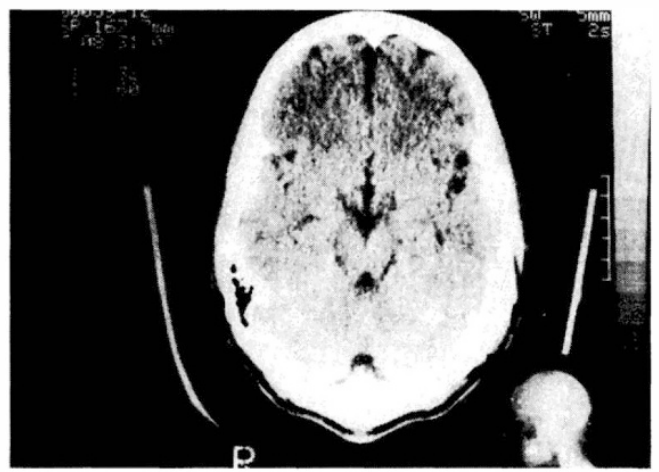

$\mathbf{b}$

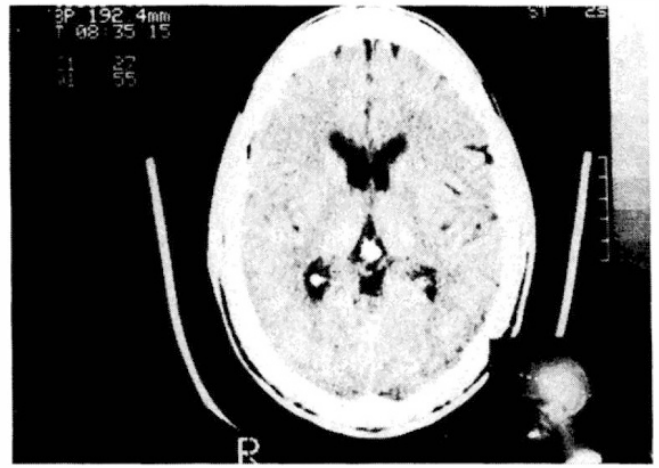

d

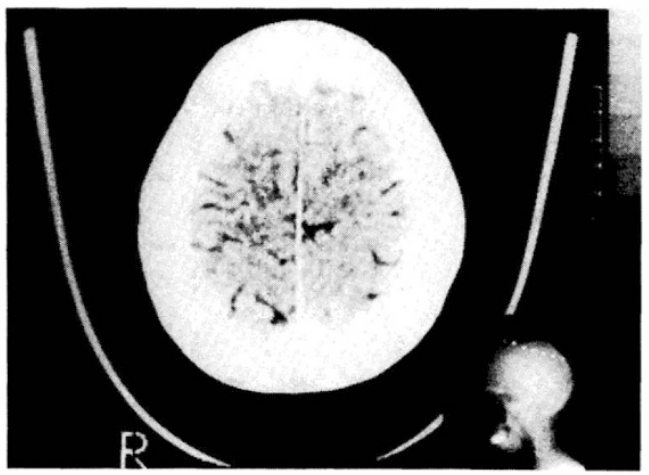

Figure 4 CT of brain: axial cuts (16 months after trauma). (a) At the level of the bodies of the lateral ventricles. Note the slight reduction in ventricular size. (b) At the level of the third ventricle. An apparent decrease in ventricle size (CVI-I 0.3 versus 0.35 in Figure $3 b$ ). (c) At the level of the fourth ventricle and lower part of the frontal lobes. Note that the temporal horns are very small. (d) At a supraventricular level. Widened sulci are demonstrated.

be a reasonable hypothesis to explain IICP. The sympathetic outflow has a role in cerebral blood flow autoregulation and CSF production. ${ }^{11}$ In animal studies, excision of the cervical sympathetic trunk increased CSF production by $30 \% .{ }^{12}$ In the present patient, however, there was no evidence of injury above the T8 level; therefore, this hypothesis is unlikely in our case.

It may also be argued that the IICP was attributable to the patient's concomitant head trauma in the same accident. Selhorst et $a l^{13}$ identified low grade papilledema in $3.5 \%$ of patients after acute severe head injury (generally within 2 weeks of trauma). They explained the papilledema either by delayed focal or diffuse cerebral swelling or by impairment of CSF absorption. Some CT findings in our patient (widening of CSF spaces and bifrontal hypodensities) constituted a possible indication of brain damage resulting from the traumatic event. ${ }^{14,15}$ However, the head trauma was not severe; it was followed by a very mild brain concussion and left no clinical signs of brain damage. Hence, its contribution to the induction of papilledema, detected 3 weeks after the trauma, is uncertain. It seems to us plausible that the more significant spinal injury was the main contributor to the IICP and the papilledema.

In conclusion, spinal injury may be followed by IICP and late papilledema. Medication is probably indicated, and the IICP and papilledema may be benign and transient if treated conservatively. Repeat 
ophthalmoscopic examinations are of clinical importance and may also reveal the true incidence of papilledema following spinal injury.

\section{References}

1 Tanaka K, Waga S, Shimoshaka S (1988) Papilledema and spinal cord tumors. Surg Neurol 29: 462-466.

2 Toulemonde V, Labrune P, Sainte Rose C, Odievre M (1989) Intermittent intracranial hypertension revealing a tumor of the cervical spine. Arch Fr Pediatr 46: 667-669.

3 Purohit AK, Dinakar I, Sundaram C, Ratankar KS (1990) Anaplastic astrocytoma of the spinal cord presenting with features of raised intracranial pressure. Child Nerv Syst 6: 113-115.

4 Firsching R, Richard KE, Thun F (1988) Acute hydrocephalus in infectious spinal disorder. Neurosurg Rev 11: $103-105$.

5 Hahn FJY, Rim K (1976) Frontal ventricular dimensions on normal computed tomography. Am J Roentg 126: 593-596.

6 Duke-Elder S (1971) System of Ophthalmology. Henry Kimpton, London: 60-65.

7 Hayreh SS (1964) Pathogenesis of oedema of the optic disc (papilledema). Br J Ophthalmol 48: 522-543.

8 Minckler DS, Tso MOM, Zimmerman LE (1976) A light microscopic autoradiographic study of axoplasmic flow in the optic nerve head during ocular hypotony, increased intraocular pressure and papilledema. $A m J$ Ophthalmol 82: 741-757.

9 Tso MOM, Hayreh SS (1977) Optic disc edema in raised intracranial pressure. IV: Axoplasmic transport in experimental papilledema. Arch Ophthalmol 95: 1458-1462.

10 Adams RD, Victor M (1985) Disturbances of cerebrospinal fluid circulation, including hydrocephalus and meningeal reactions. In: Principles of Neurology. McGraw-Hill, Singapore: 461-473.

11 Cole JD. The pathophysiology of the autonomic nervous system in spinal cord injury. In: Illis LS, editor. Spinal Cord Dysfunction: Assessment. Oxford University Press, Oxford: 201-235.

12 Edvinsson L (1982) Sympathetic control of cerebral circulation. Trends Neurosci 5: 425-429.

13 Selhorst JB, Gudeman SK, Butterworth JF, Harbison JW, Miller JD, Becker DP (1985) Papilledema after acute head injury. Neurosurgery 16: $357-363$.

14 Jennett B, Teasdale G (1981) Management of Head Injuries. FA Davis Company, Philadelphia: 19-43.

15 Reider-Grosswasser I (1991) Late CT findings in traumatic brain injury. I: Linear measurements. The International Brain Injury Symposium, New Orleans, USA, 1991. 